\title{
Bond strength of three luting agents to zirconia ceramic - Influence of surface treatment and thermocycling
}

\begin{abstract}
Ahmed ATTIA
MScD, Dr Med Dent, PhD, ,Associate Professor, Department of Conservative Dentistry and Fixed Prosthodontics, Faculty of Dentistry, Mansoura University, Mansoura, Egypt
\end{abstract}

Corresponding address: Ahmed Attia - Department of Conservative Dentistry and Fixed Prosthodontics - Faculty of Dentistry, Mansoura University - P C 35516 - P O Box 40 - Mansoura - Egypt - TPhone: +2 0502211440 - Fax: +2 0502260173 - e-mail: aattia@mans.edu.eg

Received: November 20, 2009 - Modification: April 30, 2010 - Accepted: October 26, 2010

\section{ABSTRACT}

$\mathrm{O}$ bjective: This in vitro study aimed to evaluate the influence of different surface treatments, 3 luting agents and thermocycling on microtensile bond strength ( $\mu$ TBS) to zirconia ceramic. Material and Methods: A total of 18 blocks ( $5 \times 5 \times 4 \mathrm{~mm}$ ) were fabricated from zirconia ceramic (ICE Zirkonia) and duplicated into composite blocks (Alphadent). Ceramic blocks were divided into 3 groups $(n=6)$ according to the following surface treatments: airborne-particle abrasion (AA), silica-coating, (SC) (CoJet) and silica coating followed by silane application (SCSI) (ESPE Sil). Each group was divided into 3 subgroups $(n=2)$ according to the 3 luting agents used. Resin-modified glass-ionomer cement (RMGIC, Ketac Cem Plus), self-adhesive resin cement (UN, RelyX Unicem) and adhesive resin cement (ML, MultiLink Automix) were used for bonding composite and zirconia blocks. Each bonding assembly was cut into microbars ( $10 \mathrm{~mm}$ long and $1 \pm 0.1 \mathrm{~mm}^{2}$ ). Seven specimens of each subgroup were stored in water bath at $37^{\circ} \mathrm{C}$ for 1 week. The other 7 specimens were stored in water bath at $37^{\circ} \mathrm{C}$ for 30 days then thermocycled (TC) for 7,500 cycles. $\mu$ TBS values were recorded for each specimen using a universal testing machine. Statistical analyses were performed using a 3-way ANOVA model followed by serial 1-way ANOVAs. Comparison of means was performed with Tukey's HSD test at $(\alpha=0.05)$. Results: $\mu$ TBS ranged from 16.8 to $31.8 \mathrm{MPa}$ after 1 week and from 7.3 to $16.4 \mathrm{MPa}$ after 30 days of storage in water and thermocycling. Artificial aging significantly decreased $\mu$ TBS $(p<0.05)$. Considering surface treatment, SCSI significantly increased $\mu$ TBS $(p<0.05)$ compared to SC and AA. Resin cements (UN and ML) demonstrated significantly higher $\mu$ TBS $(p<0.05)$ compared to RMGIC cement. Conclusions: Silica coating followed by silane application together with adhesive resin cements significantly increased $\mu \mathrm{TBS}$, while thermocycling significantly decreased $\mu$ TBS.

Key words: Ceramics. Surface treatment. Resins. Bond strength.

\section{INTRODUCTION}

High mechanical properties, chemical stability, and biocompatibility make zirconia an attractive core material for fabrication of all-ceramic restorations $s^{4,7,15-19}$. Bonding techniques of allceramic restorations are dependent on chemical compositions of each ceramic system ${ }^{12}$. Hydrofluoric acid etching and silanization are mandatory steps to achieve a durable resin bonding to silica ceramics ${ }^{12}$. On the other hand zirconia ceramic requires alternative techniques for long-term durable resin bonding $2,10,23$. Therefore several surface treatments are used to improve bonding to zirconia ceramic ${ }^{3,5,6}$, such as selective infiltration etching (SIE) technique ${ }^{1}$, laser etching ${ }^{8}$, alumina coating ${ }^{11}$, silica ceramic coating ${ }^{13}$, tribochemical silica coating ${ }^{3,6,9,12,22}$ or airborne-particle abrasion $3,6,9,14,20,22,25$. Several ceramic primers have been introduced into the dental market recently to enhance chemical bonding to zirconia ceramic ${ }^{12,14,16}$, such as primers containing a phosphonic acid monomer, 6-MHPA 
(6-methacryloxyhexylphosphonoacetate), (AZ, primer) or 3-trimethoxysilylpropyl methacrylate, MDP, ethanol (Clearfil Ceramic Primer) and organophosphate monomer, carboxylic acid monomer and other monomers (Z-Prime Plus) ${ }^{12,14,16}$. However, there are obvious problems in obtaining a durable bonding to zirconia ceramics ${ }^{7,14}$.

Conventional luting agents, such as glass ionomer cement (GIC), could be used for cementation of zirconia ceramic full-coverage restorations $s^{5,9,14}$. However adhesive cementation is preferred in case of compromised retention and resin-bonded fixed dental prosthesis ${ }^{3-5,14}$. Self-adhesive resin cements have been introduced into the dental market to simplify bonding procedures ${ }^{21}$. However bond strength results to zirconia ceramics in the literatures using different categories of luting agents are very controversial2,9,15,22,25. Moreover, chemical composition of zirconia ceramic and intaglio surface morphology are unique for each commercial system ${ }^{4,5}$. Therefore, conclusions drawn considering bonding to one zirconia ceramic system may not be applicable to other systems $\mathbf{s}^{4,5}$.

Recently, a new zirconia ceramic (ICE Zirconia, ZirkonZahn) has been introduced in the dental market. According to its manufacturer, this zirconia ceramic has bending strength over $1,400 \mathrm{MPa}$ and could be used for fabrication of 16-unit fixed dental prosthesis. However, no independent data considering bonding to this zirconia ceramic has been published yet.

Several studies have evaluated bond strength of adhesives in vitro in terms of shear ${ }^{4,5,18}$, tensile ${ }^{27,28}$ and microtensile bond strength ( $\mu$ TBS $)^{2,26,30}$. However, $\mu$ TBS test is considered as the most accurate one ${ }^{24,30}$. Moreover, aging and thermocycling are two important factors that have been shown to decrease the bond strength in in vitro studies ${ }^{4,5}$. The purpose of this study was to investigate in vitro the influence of different surface treatments, storage in water and thermocycling, on the $\mu$ TBS of 3 luting agents to this zirconia ceramic. The null hypotheses of the study were (1) a durable bonding to the zirconia ceramic would be achieved regardless of the surface treatments, (2) self-adhesive resin cement would provide a durable bonding to this zirconia ceramic similar to multistep adhesive resin cement, and (3) resin-modified GIC (RMGIC) would provide a durable bonding to this zirconia ceramic compared to both adhesive resin cements.

\section{MATERIAL AND MEHTODS}

A total of 18 fully-sintered zirconia blocks $(5 \times 5 \times 4 \mathrm{~mm})$ in dimension were used for this study. Each ceramic block was duplicated in lightpolymerized hybrid, type 2 restorative composite resin (Alphadent composite, shade $\mathrm{A} 2$ ) using vinyl polysiloxane material (President, Coltène Whaledent, Altstätten, Switzerland) (Figure 1). Composite resin was applied in increments 1-2 $\mathrm{mm}$ thickness and carefully condensed with ST Instrument with plastic working end (OptraSculpt, Ivoclar Vivadent, Schaan, Liechtenstein). Each increment was light-polymerized for $40 \mathrm{~s}$ at $5 \mathrm{~mm}$ distance and an intensity of irradiation $130 \mathrm{~mW} / \mathrm{cm}^{2}$ (FutoLux 2, Carlo De Gorgi, Milano, Italy).

\section{Surface treatment before bonding}

Ceramic blocks were divided into 3 groups $(n=6)$ according to surface treatment as follow: Group 1: airborne-particle abrasion (AA), using $\mathrm{Al}_{2} \mathrm{O}_{3}(50-\mu \mathrm{m}$ aluminum oxide particles, Pluradent, Offenbach, Germany) at $0.28 \mathrm{MPa}$ for $13 \mathrm{~s}$ at a distance of 10 $\mathrm{mm}^{4}$ (Ney Blastmate II, Ney, CA, USA). Zirconia ceramic blocks were ultrasonically cleaned in distilled water for $3 \mathrm{~min}$ and dried with oil-free air stream; Group 2: Silica coating (SC), airborneparticle abrasion using $50-\mu \mathrm{m} \mathrm{Al}_{2} \mathrm{O}_{3}$ particles at 0.28 $\mathrm{MPa}$ for $13 \mathrm{~s}$ at a distance of $10 \mathrm{~mm}^{4}$ followed by airborne-particle abrasion with $30-\mu \mathrm{m} \mathrm{SiO}_{2}$ particles (CoJet sand, 3M ESPE, Seefeld, Germany) at a pressure of $0.25 \mathrm{MPa}$ for $20 \mathrm{~s}$ at a distance of 10 $\mathrm{mm}^{26}$ (Ney Blastmate II). Zirconia ceramic blocks were ultrasonically cleaned in distilled water for $3 \mathrm{~min}$ and dried with oil-free air stream; Group 3 Silica coating and silane application (SCSI), same as Group SC followed by silane application ${ }^{26}$ (ESPE Sil, 3-methacryloxyprophyltrimethoxysilane in ethanol, 3M ESPE). Five minutes were allowed to elapse for silane reaction ${ }^{26}$.

\section{Bonding zirconia and composite blocks}

Each main group was divided into 3 subgroups $(n=2)$ according to the following 3 luting agents, Ketac Cem Plus, RelyX Unicem and MultiLink Automix. Bonding procedures were performed according to the manufacturers' recommendations for each luting agent:

Ketac Cem Plus (GI): self curing, radiopaque, fluoride-releasing, RMGIC containing BisGMA and HEMA (3M ESPE). Equal amounts of past $A$ and $B$ were extruded on waxed paper pad, mixed for $20 \mathrm{~s}$ using a plastic spatula until a uniform color was achieved. The mix was applied to the intaglio surfaces of the zirconia ceramic blocks.

RelyX Unicem Aplicap (UN): dual-cure, selfadhesive resin cement, containing phosphoric acid monomer and methacrylate monomers (3M ESPE). The capsule was activated for $4 \mathrm{~s}$, and then mixed in amalgamator (Silver Mix 80, Carlo De Giorgi, Milano, Italy) for $10 \mathrm{~s}$. The capsule was inserted into the applier and cement was dispensed directly onto the intaglio surfaces of the zirconia ceramic blocks.

MultiLink Automix (ML): self-curing, transparent, two-past adhesive resin cement, containing 
dimethacrylate and HEMA (Ivoclar Vivadent). Equal amounts of adhesive resin cement were extruded, mixed for $20 \mathrm{~s}$ and applied to the intaglio surfaces of the zirconia ceramic blocks. Finally composite blocks were bonded to intaglio surfaces of zirconia ceramic blocks. Excess luting cement was removed using disposable minibrush (Ivoclar Vivadent). The bonding assembly was kept under a static load of $40 \mathrm{~N}$ for 5 min (Articolo 719/00, \#1L01; Carlo De Giorgi), Combination of 3 surface treatments and 3 luting agents resulted in 9 test groups as follows: GIAA, GISC, GISCSI, UNAA, UNSC, UNSCSI, MLAA, MLSC and MLSCSI.

\section{Preparation of microbars}

Each block was then bonded with cyanoacrylate glue (Uhu ${ }^{\circledR}$, batch 40267647, Uhu, Bühl, Germany) to a metal base that was fixed to a cutting machine ${ }^{2,26}$ (Isomet 1000, Buehler Ltd., Lake Bluff, IL, USA). Cutting was created under wet condition. The first 0.5-mm-thick slice was discarded $2,6,26$. Three slices $1 \pm 0.1 \mathrm{~mm}$ in thickness were obtained. Each slice was rotated $90^{\circ}$ and bonded to another metal base again $2,6,26$. The first slice $0.5 \mathrm{~mm}$ was also disregarded. Other 3 additional cuts $1 \pm 0.1$ $\mathrm{mm}$ in thickness were made ${ }^{2,6,26}$. This procedure was repeated for the other 2 slices. A total of 9 non-trimmed bar specimens $(10 \mathrm{~mm}$ long and $1 \pm 0.1 \mathrm{~mm}^{2}$ ) bonded surface area were obtained from each block ${ }^{6}$. Microscopic examination (Wild Makroskop M 420; Heerbrugg, Switzerland) at $20 \times$ magnification of the bar specimens revealed that only 15 specimens from group GISC and 14 specimens from group GISCSI were free from microcracks. Therefore for standardization of the test groups, only 14 specimens from each subgroup were used to complete the test. Seven specimens from each subgroup were stored in water bath at $37^{\circ} \mathrm{C}$ for 1 week. While the other 7 specimens were stored in water bath at $37^{\circ} \mathrm{C}$ for one month followed by thermocycling (TC) for 7,500 cycles. Each cycle was consists of 1 minute in $5^{\circ} \mathrm{C}$ cold bath and 1 minute in $55^{\circ} \mathrm{C}$ hot bath with a dwell time of $30 \mathrm{~s}$. Specimens were dried and glued parallel to the long axis of an adapted caliper using cyanoacrylate glue (Uhu ${ }^{\circledR}$, batch 40267647, Uhu). This apparatus was fixed to the universal testing machine (Type 500, Lloyd Instrument, Farnham, UK).

\begin{tabular}{|c|c|c|}
\hline Materials & Lot/Batch No & Manufacturer \\
\hline $\begin{array}{l}\text { Zirconium-oxide ceramic with the following composition }\left[\mathrm{Y}_{2} \mathrm{O}_{3} \text {, }\right. \\
\text { 4.95-5.26, } \mathrm{Al}_{2} \mathrm{O}_{3}, 0.15-0.35, \mathrm{SiO}_{2}, \mathrm{Max} 0.02, \mathrm{Fe}_{2} \mathrm{O}_{3} \max \text { 0.01, } \\
\left.\qquad \mathrm{Na}_{2} \mathrm{O}_{3} \mathrm{Max} .0 .04\right]\end{array}$ & CE0476 & $\begin{array}{l}\text { ICE Zirkonia, ZirkonZahn, Gais, } \\
\text { Italy }\end{array}$ \\
\hline $\begin{array}{c}\text { Transparent, two past, self curing adhesive resin cement } \\
\text { dimethacrylate and HEMA, with barium glass silica and filler } \\
\text { base/catalyst past in Clicker }\end{array}$ & K49940 & $\begin{array}{c}\text { Multilink Automix; Ivoclar } \\
\text { Vivadent, Schaan, Liechtenstein }\end{array}$ \\
\hline $\begin{array}{l}\text { Self curing, radiopaque, fluoride-releasing, resin modified glass } \\
\text { ionomer cement } \\
\text { Paste A/Paste B in Clicker } \\
\text { Paste A, fluoroaluminosilicate (FAS) glass, proprietary reducing } \\
\text { agent, HEMA, water, opacifying agent } \\
\text { Paste B, methacrylated polycarboxylic acid, BisGMA, HEMA, } \\
\text { water, potassium persulfate, zirconia silica filler }\end{array}$ & 56930/PF8ML & $\begin{array}{l}\text { Ketac Cem Plus,3M ESPE, } \\
\text { Seefeld, Germany }\end{array}$ \\
\hline $\begin{array}{l}\text { Dual-curing, universal self adhesive resin cement in capsule, } \\
\text { Liquid, methacrylate monomers containing phosphoric acid } \\
\text { groups, methacrylate monomers, Initiator components, } \\
\text { Stabilizers } \\
\text { Powder, alkaline (basic) fillers, silanated fillers, initiator } \\
\text { components, pigments }\end{array}$ & 325464 & $\begin{array}{l}\text { RelyX Unicem Aplicap, 3M ESPE, } \\
\text { Seefeld, Germany }\end{array}$ \\
\hline $\begin{array}{l}\text { Silane coupling agent } \\
\text { 3-methacryloxyprophyltrimethoxysilane in ethanol }\end{array}$ & 323995 & ESPE Sil, 3M ESPE \\
\hline $\begin{array}{l}50 \mu \mathrm{m} \mathrm{Al}_{2} \mathrm{O}_{3} \text { particles for } \\
\text { airborne-particle abrasion } \\
\qquad 30 \mu \mathrm{m} \mathrm{SiO}_{2}\end{array}$ & 14540 & $\begin{array}{c}\text { Alumina particles } \\
\text { Pluradent, Offenbach, } \\
\text { Germany } \\
\text { CoJet - Sand, 3M ESPE }\end{array}$ \\
\hline Visible light activated, radiopaque hybrid, type 2 composite resin & P121FJ & $\begin{array}{l}\text { Alpha.Dent composite, Dental } \\
\text { technologies, Illinois, USA }\end{array}$ \\
\hline
\end{tabular}

Figure 1- Materials used in the study 
Microtensile bond strength test

Specimens were loaded in tension to failure (Figure 2) at a crosshead speed of $1 \mathrm{~mm} / \mathrm{min}$. Microtensile bond strength $\mu$ TBS values were recorded for each specimen in $\mathrm{MPa}$ using the formula:

$\alpha=L / A$, where ' $L$ ' is the load at failure $(N)$ and ' $A$ ' is the bonded area $1 \pm 0.1 \mathrm{~mm}^{2}$.

Statistical analysis was conducted using the SPSS statistical software, version 16.0 (SPSS Inc, Chicago, Ill, USA). Statistical analyses were performed with 3-way ANOVA model followed by serial 1-way ANOVAs. Post Hoc Tukey-HSD test at $\alpha=0.05$ was performed to test statistical significance between the groups.

\section{Microscopic examination of the debonded specimens}

The fractured interfaces of the debonded specimens were examined with a light microscope (Wild Makroskop M 420) at x20 magnification to determine the failure pattern, which was assigned to cohesive failure within resin cement or composite resin ${ }^{28}$, adhesive at ceramic/cement interface ${ }^{28}$ or

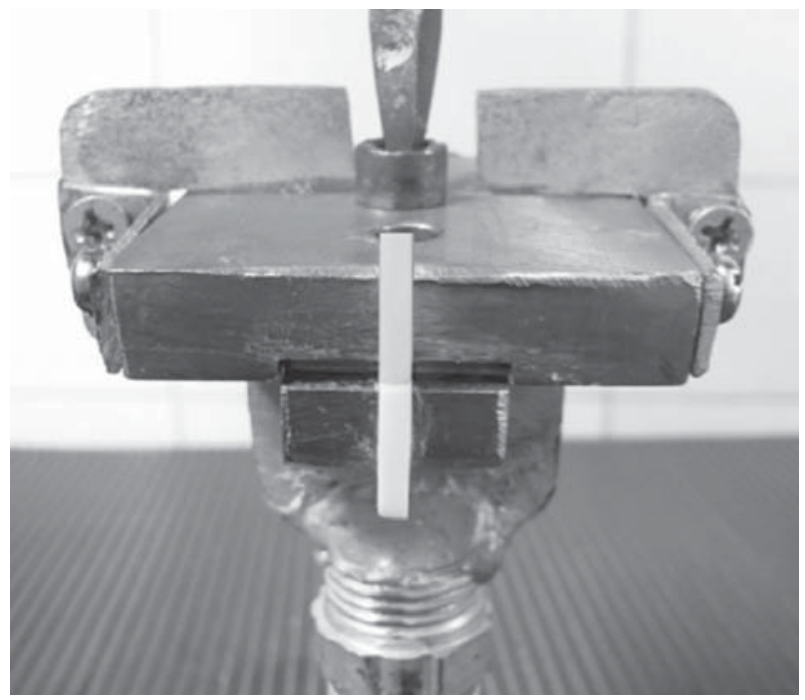

Figure 2- Debonded specimen glued to an adapted caliper and fixed to the universal testing machine

Table 1- Mean \pm standard deviations (SD), minimum and maximum microtensile bond strength in MPa after 1 week and 1 month storage in water with thermocycling of test groups and $P$ values

\begin{tabular}{|c|c|c|c|c|c|}
\hline \multirow[t]{2}{*}{ Test groups } & \multicolumn{2}{|c|}{ One week } & \multirow{2}{*}{$\begin{array}{c}\text { One month/ } \\
\text { Thermocycling } \\
\text { Mean士SD }\end{array}$} & \multirow[b]{2}{*}{ Min. } & \multirow[b]{2}{*}{$P$ values } \\
\hline & Mean $\pm S D$ & Min. & & & \\
\hline & & Max. & & Max. & 1 week Vs 1 month/TC \\
\hline \multirow[t]{2}{*}{ GIAA } & $18 \pm 4.3$ & 11.4 & $7.3 \pm 3.5$ & 2.3 & $<.001^{*}$ \\
\hline & & 23.5 & & 12.8 & \\
\hline \multirow[t]{2}{*}{ GISC } & $17.3 \pm 3.9$ & 11.6 & 0 & - & Debonded \\
\hline & & 22 & & & \\
\hline \multirow[t]{2}{*}{ GISCSI } & $16.8 \pm 3.7$ & 13.3 & 0 & - & Debonded \\
\hline & & 23 & & & \\
\hline \multirow[t]{2}{*}{ MLAA } & $18.7 \pm 2.5$ & 14.8 & $10 . \pm 2.4$ & 7.1 & $.004^{*}$ \\
\hline & & 22.7 & & 13.7 & \\
\hline \multirow[t]{2}{*}{ MLSC } & $20.4 \pm 2.2$ & 17.1 & $9.7 \pm 3$ & 6.1 & $<.001^{*}$ \\
\hline & & 23.7 & & 15 & \\
\hline \multirow[t]{2}{*}{ MLSCSI } & $31.8 \pm 3.4$ & 26.6 & $15.4 \pm 2.8$ & 11.3 & $<.001^{*}$ \\
\hline & & 36.3 & & 18.6 & \\
\hline \multirow[t]{2}{*}{ UNAA } & $19.1 \pm 4.4$ & 11.9 & $9.2 \pm 3.9$ & 6.2 & $.04^{*}$ \\
\hline & & 23.8 & & 18.6 & \\
\hline \multirow[t]{2}{*}{ UNSC } & $21.6 \pm 3.3$ & 17.3 & $13.1 \pm 3.8$ & 8.4 & $.006^{*}$ \\
\hline & & 26.2 & & 19.2 & \\
\hline \multirow[t]{2}{*}{ UNSCSI } & $28.6 \pm 6.2$ & 19.2 & $16.4 \pm 4.2$ & 10.5 & $<.001^{\star}$ \\
\hline & & 36 & & 24 & \\
\hline
\end{tabular}

Group codes

Gl: Ketac Cem Plus

ML: Multilink Automix

UN: RelyX Unicem
AA: Air borne particle abrasion

SC: Silica coating

SCSI: Silica coating and silane application 
ATTIA A

\begin{tabular}{|l|c|c|c|c|c|c|}
\hline $\begin{array}{c}\text { "Test groups } \\
\text { Failure Pattern" }\end{array}$ & \multicolumn{4}{|c|}{ One week } & \multicolumn{3}{c|}{ One month/Thermalcycling } \\
\hline & Mixed & Adhesive & Cohesive & Mixed & Adhesive & Cohesive \\
\hline MLSCSI & 4 & 3 & 0 & 2 & 5 & 0 \\
\hline MLSC & 2 & 3 & 2 & 1 & 6 & 0 \\
\hline MLAA & 3 & 3 & 1 & 0 & 7 & 0 \\
\hline UNSCSI & 3 & 4 & 0 & 2 & 5 & 0 \\
\hline UNSC & 6 & 0 & 1 & 2 & 5 & 0 \\
\hline UNAA & 3 & 4 & 0 & 2 & 5 & 0 \\
\hline GISCSI & 1 & 5 & 1 & 0 & 7 & 0 \\
\hline GISC & 3 & 3 & 1 & 0 & 7 & 0 \\
\hline GIAA & 3 & 4 & 0 & 1 & 6 & 0 \\
\hline
\end{tabular}

Group codes
GI: Ketac Cem Plus
AA: Air borne particle abrasion
ML: Multilink Automix
SC: Silica coating
UN: RelyX Unicem
SCSI: Silica coating and silane application

Figure 3- Failure pattern of all test groups after 1 week and 1 month storage in water

mixed adhesive/cohesive modes ${ }^{28}$. Representative specimens for each failure pattern were examined using a scanning electron microscope (SEM; XL $30 \mathrm{CP}$; Philips, Eindhoven, Netherlands) with an acceleration voltage of $15 \mathrm{kV}$ and a working distance of $10 \mathrm{~mm}$.

\section{RESULTS}

Mean \pm standard deviation, minimum and maximum $\mu$ TBS without and with thermocycling of test groups in MPa and P values are summarized in (Table 1). $\mu$ TBS means were compared across all test groups using 3-way ANOVA model including the following factors (luting agent, surface treatment, storage time and interaction). The overall F-test was highly significant $(p<0.0001)$, indicating differences in mean $\mu$ TBS across at least one of the 3 factors. All individual factors were significant, $(p<0.001)$. The interaction between surface treatment and luting agent and surface treatment and storage time were significant $(P<0.001)$. However luting agent and storage time was not significant $(p=0.208)$.

Multiple comparisons with Post Hoc Tukey-HSD test at $a=0.05$ revealed that, considering 3 surface treatment performed, SCSI significantly increased $\mu$ TBS $(p<0.05)$ compared to SC and AA. However, there was no statistically significant difference between SC and AA ( $p=0.26)$.

Considering 3 luting agents used, ML and UN resin cements showed significantly higher $\mu$ TBS than the GIC cement $(p<0.001)$. However, there was no significant difference in the $\mu$ TBS of the 2 resin cements $(p=0.37)$.

Considering aging condition, 30-day water storage and thermocycling significantly decreased

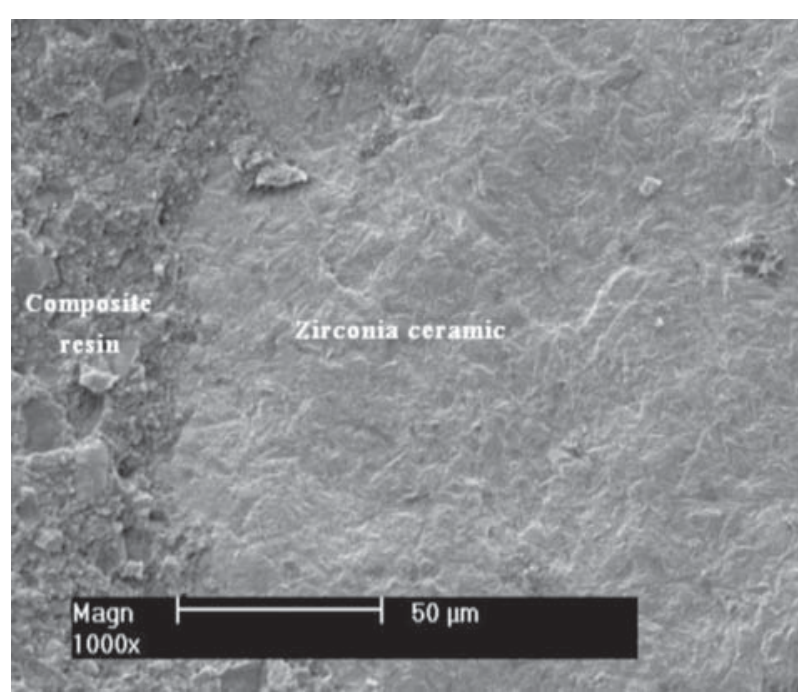

Figure 4- Representative scanning electron microscope micrograph at 1,000x magnification showing mixed failure, adhesive at ceramic/cement interface and cohesive within the composite resin restorative material

$\mu$ TBS compared to 1 -week water storage $(p<0.05)$. Groups GISC and GISCSI were spontaneously debonded.

Fracture patterns of all test groups are summarized in (Figure 3). After 1 week of storage in water, the debonded specimens showed mainly mixed failure pattern (Figure 4). Some specimens showed adhesive and cohesive failure (Figure 5). After 30 days of storage in water and thermocycling, the failure pattern was mainly adhesive with remnants of the luting cements still adhered to zirconia ceramic surface (Figure 6). 


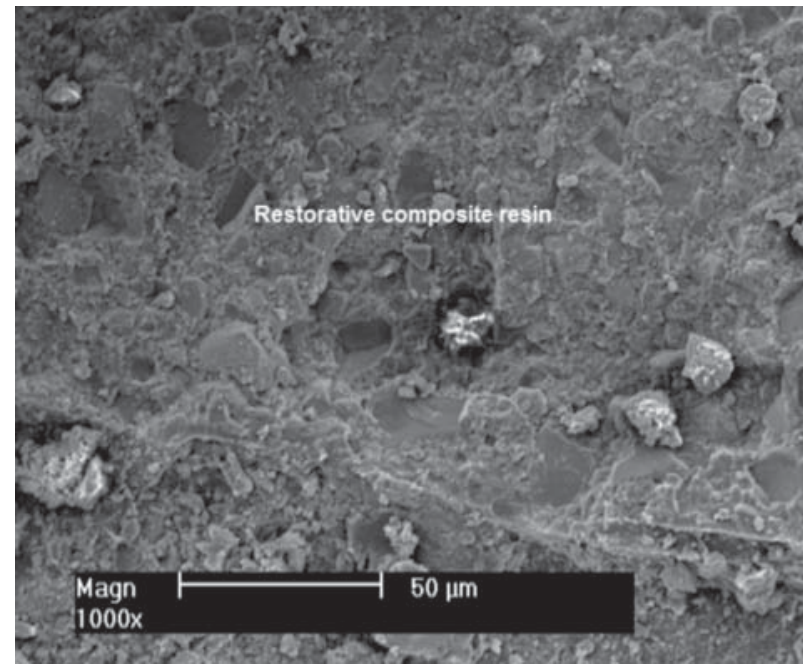

Figure 5- Representative scanning electron microscope micrograph at $1,000 x$ magnification showing cohesive failure within the composite resin restorative material

\section{DISCUSSION}

Clinically, restorations are subjected to repeated thermal stress and mechanical fatigue due to masticatory forces. Therefore, one limitation of this study is that specimens were subjected only to thermal stress without mechanical fatigue. In addition, storage in water during 1 month might be too short to allow water saturation of the luting cements. It is likely that hydrolytic effects might affect the bond strength negatively after longer time of storage in water.

Microtensile bond strength test is more accurate than shear and tensile2,30. Because the small dimensions and small interfacial bonding zone of the specimens result in a more uniform distribution of the applied stresses ${ }^{2,10,30}$. Airborne-particle abrasion and silica coating are surface treatments $s^{4,22,26}$ recommended by most of the manufactures of zirconia ceramics and luting agents to improve bonding to zirconia ceramics. Therefore, these surface treatments were tested in this study.

A range of $10-13 \mathrm{MPa}$ was suggested as the minimum range for acceptable clinical bonding ${ }^{15}$. Therefore the results of this study clearly indicated that, silica coating and silane application together with resin cements would ensure a durable resin bonding to this zirconia ceramic 15-16 Mpa.

Considering surface treatment, airborne-particle abrasion produced an activated microroughened zirconia surface, increased the bonding area and modifying the surface energy and wettability 21,22 . In case of silica coating, alumina particles modified with silica acid were sprayed under pressure ${ }^{10,29}$. This tribochemical reaction produces a high temperature contact area that can hold the silica

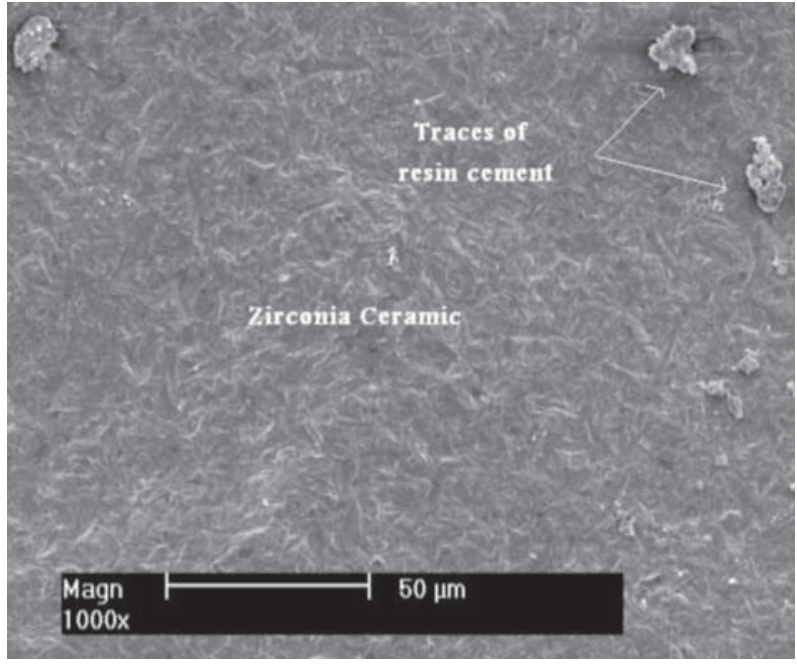

Figure 6- Representative scanning electron microscope micrograph at 1,000x magnification showing adhesive failure at ceramic/cement interface

layer on the ceramic surface $3,6,10,29$. These particles formed a base for micromechanical interlocking ${ }^{10}$. Because no silane was applied before bonding, bond strength was directly correlated to the quality of micromechanical interlocking with the silica coating layer. However, bond strength to zirconia ceramic after silica coating was not improved in comparison to airborne-particle abrasion.

The results of this study are in agreement with the findings of several studies $21,22,25$, which reported that bond strength to zirconia ceramics was not improved after silica coating compared to airborne-particle abrasion. Oyagüe, et al.21,22 (2009) and Kern ${ }^{12}$ (2009) reported that airborneparticle abrasion of zirconia ceramics produces a certain roughness, but only limited undercuts were produced, thus not improving bonding to zirconia. Matinlinna, et al. ${ }^{18}$ (2006), after energydispersive $\mathrm{x}$-ray analysis of silica-coated zirconia ceramic, reported that silica-coverage originating from the coating particles appears not to have become embedded onto the hard zirconia surface, consequently bond strength was not improved after silica coating. Moreover, ultrasonic cleaning in distilled water might removed a significant amount of silica coating layer, consequently bond strength of resin cements to silica-coated zirconia ceramic was decreased, as reported by Nishigawa, et al. ${ }^{19}$ (2008). However, the results of this study were contradicting to the results of other studies ${ }^{3,15}$, which reported that silica coating improved bond strength to zirconia ceramics compared to airborneparticle abrasion. This difference in the results could be attributed to the fact that, Atsu, et al. ${ }^{3}$ (2006) used $125-\mathrm{mm} \mathrm{Al}_{2} \mathrm{O}_{3}$ for airborne-particle abrasion followed by $30-\mathrm{mm} \mathrm{Al}_{2} \mathrm{O}_{3}$ particles modified by silica. Panavia luting cement together with different ceramic primers were used for bonding. On the 
other hand Lüthy, et al. ${ }^{15}$ (2006) used the Rocetac system for silica coating, which combines $110 \mathrm{~mm}$ $\mathrm{Al} 2 \mathrm{O} 3$ for airborne-particle abrasion followed by 110- $\mathrm{mm} \mathrm{Al}_{2} \mathrm{O}_{3}$ particles modified by silica. Rounded rods were bonded to zirconia ceramics instead of restorative composite resin. Moreover both studies used shear test. In the present study, 50-mm Al${ }_{2} \mathrm{O}_{3}$ was used for airborne-particle abrasion followed by 30-mm Al $\mathrm{O}_{3}$ particles modified by silica and a $\mu$ TBS test were employed.

Silica coating and silane application significantly increased bond strength compared to airborneparticle abrasion or silica coating alone, as reported in several studies ${ }^{2,3,10}$. Silane coupling agent did not promote adequate bonding to zirconia ceramics, as these ceramics contain minimal or no silica content ${ }^{9,12,14}$. Therefore, silica coating of zirconia ceramics is a prerequisite for durable siloxane bonding, as it leave a physically and chemically active outer surface layer ${ }^{2,18}$. Silane coupling agent wets the adherent, increases its surface energy and makes it accessible for effective bonding ${ }^{18}$. Moreover, it is capable of forming covalent bond at silica coated ceramic/resin cement interface through formation of silanol groups ${ }^{18,26,29}$. Therefore, after silica coating and silanization, bond strength was based on both micromechanical interlocking plus chemical adhesion due to silane application 18,26,29.

Variations in chemical composition, wetting capacity, viscosity and mechanical properties for each luting cement could be responsible for variations in the bonding capacity to zirconia ceramics $915,17,20,28$. According to the manufacturer, RelyX Unicem contains methacrylate monomers, adhesive phosphate monomer and silanated fillers in its chemical composition. Adhesive phosphate monomer enhanced self bonding to zirconia ceramics especially after silica coating and silane application as reported in several studies ${ }^{12,15,20}$. Ketac Cem Plus contains BisGMA, HEMA and zirconia filler. Several studies have reported that bond strengths of BisGMA containing resin cements were dramatically decreased after artificial aging ${ }^{12,15}$ due to their remarkable weak mechanical properties.

Multilink Automix contains no adhesive phosphate monomer. However, it contains dimethacrylate, HEMA and silica filler. Dimethacrylate and HEMA improved its mechanical properties. The improved mechanical properties could be responsible for high bond strength results of Multilink Automix. Nothdurft, et al. ${ }^{20}$ (2009) found no significance difference in the mean bond strength of Multilink Automix 21.2 MPa and RelyX Unicem 23.1 MPa to zirconia ceramics after different surface treatments.

RelyX Unicem and Multilink Automix have better mechanical properties than Ketac Cem Plus. According to the manufacturers flexural strength for RelyX Unicem (75 MPa) and Multilink Automix
(70 $\mathrm{MPa}$ ) are in the same range. However it is higher than that of Ketac Cem Plus (31.6 MPa). Flexural strength is an indicator for the mechanical properties of each luting agent. Luting cements with high mechanical properties are more resistant to aging conditions ${ }^{15}$. Therefore, variations in the mechanical properties of the 3 luting cements used could be another contributing factor for bond strength results as reported in other studies ${ }^{15,17}$.

In in vitro studies, water storage and thermocycling are two important factors that decrease the bond strength $4,26,27$. Therefore, 1 month storage in water and thermal cycling for 7,500 cycles was used as aging regime to simulate clinical conditions. After 30 days of storage in water and thermocycling, $\mu$ TBS were significantly decreased in all groups. This decrease in bond strength might be due to degradation of the luting cement itself $f^{4,17,27}$ and the hydrolytic effect of water at the luting cement/ceramic interface ${ }^{9,27}$.

Moreover, mismatch between the coefficient of thermal expansion of the bonded specimens (zirconia ceramic, luting agent and composite resin) could result in hoop stress during thermocycling ${ }^{27}$. Another factor could be the fact that silanized surfaces were unstable in contact with moisture as reported by Derand, et al. ${ }^{9}$ (2005). Accumulation of negative effect of water, thermocycling and instability of silane could be responsible for the decrease of bond strength for all test groups.

The initial high bond strength results were reflected on the failure pattern of debonded specimens as examined by optical reflection microscope and confirmed by scanning electron microscopy. All groups showed mainly mixed failure pattern or adhesive failure while cohesive failure was minimal. Cohesive failure within composite resin could be due to the initiation of microcracks during cutting of the specimens ${ }^{24}$. Therefore, in this study, specimens with apparent microcracks under stereomicroscopic examination were discarded.

After 30 days of storage in water and thermocycling, the failure pattern was mainly adhesive indicating a decrease in the bond strength due to the hydrolytic effect of water, hoop stress due to thermocycling and degradation of the luting resin itself.

The general outcome of this study suggests that although conventional cements could be used for cementation of zirconia ceramics, resin cements are preferred for long-term bond durability. Bonding techniques based on micromechanical interlocking and chemical adhesion using silanes are preferred than other techniques. 


\section{CONCLUSIONS}

Within the limitations of this study, the following conclusions were drawn:

Silica coating and silane application significantly improved $\mu$ TBS compared to silica coating or airborne-particle abrasion.

There was no significance difference in the bond strength after using self-adhesive resin cement or multistep adhesive resin cement.

Both adhesive resin cements significantly increased $\mu$ TBS compared to RMGIC.

\section{ACKNOWLEDGMENT}

The author thanks Prof. Dr. Hassan Soltan, Faculty of Engineering, Mansoura University for his help with statistical analysis of the results. The author thanks Mr. F. Lehmann Dipl.-Ing., Department of Prosthodontics, Propaedeutics and Dental Materials, School of Dentistry, ChristianAlbrechts University at Kiel, Germany for his help with SEM micrograph. The author also thanks Ivoclar Vivadent and 3M ESPE for supplying all the materials with no cost.

\section{REFERENCES}

1- Aboushelib MN, Kleverlaan CJ, Feilzer AJ. Selective infiltrationetching technique for a strong and durable bond of resin cements to zirconia-based materials. J Prosthet Dent. 2007;98:379-88.

2- Amaral R, Ozcan M, Bottino MA, Valandro LF. Microtensile bond strength of a resin cement to glass infiltrated zirconiareinforced ceramic: the effect of surface conditioning. Dent Mater. 2006;22:283-90.

3- Atsu SS, Kilicarslan MA, Kucukesmen HC, Aka PS. Effect of zirconium-oxide ceramic surface treatments on the bond strength to adhesive resin. J Prosthet Dent. 2006;95:430-6.

4- Blatz MB, Chiche $G$, Holst $S$, Sadan $A$. Influence of surface treatment and simulated aging on bond strengths of luting agents to zirconia. Quintessence Int. 2007;38:745-53.

5- Blatz MB, Sadan A, Martin J, Lang B. In vitro evaluation of shear bond strengths of resin to densely-sintered high-purity zirconium-oxide ceramic after long-term storage and thermal cycling. J Prosthet Dent. 2004;91:356-62.

6- Bottino MA, Valandro LF, Scotti R, Buso L. Effect of surface treatments on the resin bond to zirconium-based ceramic. Int J Prosthodont. 2005;18:60-5.

7- Cehreli MC, Kökat AM, Akça K. CAD/CAM Zirconia vs. slip-cast glass-infiltrated Alumina/Zirconia all-ceramic crowns: 2-year results of a randomized controlled clinical trial. J Appl Oral Sci. 2009;17:49-55.

8- Cavalcanti AN, Foxton RM, Watson TF, Oliveira MT, Giannini M, Marchi GM. Bond strength of resin cements to a zirconia ceramic with different surface treatments. Oper Dent. 2009;34:280-7. 9- Derand T, Molin M, Kvam K. Bond strength of composite luting cement to zirconia ceramic surfaces. Dent Mater. 2005;21:115862.

10- Della Bona A, Borba M, Benetti P, Cecchetti D. Effect of surface treatments on the bond strength of a zirconia-reinforced ceramic to composite resin. Braz Oral Res. 2007;21:10-5.
11- Jevnikar P, Krnel K, Kocjan A, Funduk N, Kosmač T. The effect of nano-structured alumina coating on resin-bond strength to zirconia ceramics. Dent Mater. 2010;29:688-96.

12- Kern M. Resin bonding to oxide ceramics for dental restorations. J Adhes Sci Technol. 2009;23:1097-111.

13- Kitayama S, Nikaido T, Maruoka R, Zhu L, Ikeda M, Watanabe $A$, et al. Effect of an internal coating technique on tensile bond strengths of resin cements to zirconia ceramics. Dent Mater J. 2009;28:446-53.

14- Kitayama S, Nikaido T, Takahashi R, Zhu L, Ikeda M, Foxton $\mathrm{RM}$, et al. Effect of primer treatment on bonding of resin cements to zirconia ceramic. Dent Mater. 2010;26:426-32.

15- Lüthy $\mathrm{H}$, Loeffel $\mathrm{O}$, Hammerle $\mathrm{CH}$. Effect of thermocycling on bond strength of luting cements to zirconia ceramic. Dent Mater. 2006;22:195-200.

16- Magne $\mathrm{P}$, Paranhos MP, Burnett LH Jr. New zirconia primer improves bond strength of resin-based cements. Dent Mater. 2010;26:345-52.

17- Marchan S, Coldero L, Whiting R, Barclay S. In vitro evaluation of the retention of zirconia-based ceramic posts luted with glass ionomer and resin cements. Braz Dent J. 2005;3:213-7.

18- Matinlinna JP, Heikkinen T, Ozcan M, Lassila LV, Vallittu PK. Evaluation of resin adhesion to zirconia ceramic using some organosilanes. Dent Mater. 2006;22:824-31.

19- Nishigawa G, Maruo Y, Irie M, Oka M, Yoshihara K, Minagi S. et al. Ultrasonic cleaning of silica-coated zirconia influences bond strength between zirconia and resin luting material. Dent Mater J. $2008 ; 27: 842-8$.

20- Nothdurft FP, Motter PJ, Pospiech PR. Effect of surface treatment on the initial bond strength of different luting cements to zirconium oxide ceramic. Clin Oral Investig. 2009;13:229-35. 21- Oyagüe RC, Monticelli F, Toledano M, Osorio E, Ferrari M, Osorio R. Effect of water aging on microtensile bond strength of dual-cured resin cements to pre-treated sintered zirconium-oxide ceramics. Dent Mater. 2009;25:392-9.

22- Oyagüe RC, Monticelli F, Toledano M, Osorio E, Ferrari M, Osorio $R$. Influence of surface treatments and resin cement selection on bonding to densely-sintered zirconium-oxide ceramic. Dent Mater. 2009;25:172-9.

23- Phark JH, Duarte S Jr, Blatz M, Sadan A. An in vitro evaluation of the long-term resin bond to a new densely sintered high-purity zirconium-oxide ceramic surface. J Prosthet Dent. 2009;101:2938.

24- Scherrer SS, Cesar PF, Swain MV. Direct comparison of the bond strength results of the different test methods: a critical literature review. Dent Mater. 2010;26:e78-93.

25- Uo M, Sjögren G, Sundh A, Goto M, Watari F, Bergman M. Effect of surface condition of dental zirconia ceramic (Denzir) on bonding. Dent Mater J. 2006;25:626-31.

26- Valandro LF, Ozcan M, Amaral R, Leite FP, Bottino MA. Microtensile bond strength of a resin cement to silica-coated and silanized In-Ceram Zirconia before and after aging. Int J Prosthodont. 2007;20:70-2.

27- Wegner SM, Gerdes W, Kern M. Effect of different artificial aging conditions on ceramic-composite bond strength. Int J Prosthodont. 2002;15:267-72.

28- Wolfart M, Lehmann F, Wolfart S, Kern M. Durability of the resin bond strength to zirconia ceramic after using different surface conditioning methods. Dent Mater. 2007;23:45-50.

29- Xible AA, Jesus Tavarez RR, Araujo Cdos R, Bonachela WC. Effect of silica coating and silanization on flexural and compositeresin bond strengths of zirconia posts: an in vitro study. J Prosthet Dent. 2006;95:224-9.

30- Yesil ZD. Microtensile bond strength testing of resin cements. Inter Journal Adhes Adhes. 2009;29:352-5. 